\title{
A Conditioning Lesion Protects Axons from Degeneration via the Wallenda/DLK MAP Kinase Signaling Cascade
}

\author{
Xin Xiong and Catherine A. Collins \\ Department of Molecular, Cellular and Developmental Biology, University of Michigan, Ann Arbor, Michigan 48109-1048
}

\begin{abstract}
Axons are vulnerable components of neuronal circuitry, and neurons are equipped with mechanisms for responding to axonal injury. A highly studied example of this is the conditioning lesion, in which neurons that have been previously injured have an increased ability to initiate new axonal growth (Hoffman, 2010). Here we investigate the effect of a conditioning lesion on axonal degeneration, which occurs in the distal stump after injury, and also occurs in neuropathies and neurodegenerative disorders (Coleman, 2005). We found that Drosophila motoneuron axons that had been previously injured had an increased resiliency to degeneration. This requires the function of a conserved axonal kinase, Wallenda (Wnd)/DLK, and a downstream transcription factor. Because axonal injury leads to acute activation of Wnd (Xiong et al., 2010), and overexpression studies indicate that increased Wnd function is sufficient to promote protection from degeneration, we propose that Wnd regulates an adaptive response to injury that allows neurons to cope with axonal stress.
\end{abstract}

\section{Introduction}

When the continuity of an axon is disrupted, the distal stump degenerates through a regulated self-destruction process called Wallerian degeneration, whose cellular mechanism is poorly understood. Recent studies suggest that a conserved dileucine zipper kinase, DLK, plays a role in this degeneration process (Miller et al., 2009; Ghosh et al., 2011). However, this kinase has also been found to promote regenerative growth of injured axons (Hammarlund et al., 2009; Itoh et al., 2009; Yan et al., 2009; Ghosh-Roy et al., 2010; Xiong et al., 2010).

To further understand the relationship of these prodegenerative and pro-regenerative functions, we investigated the role of the Drosophila homolog of DLK, Wallenda (Wnd) in Wallerian degeneration of Drosophila motoneurons and synapses. We have previously described how Wnd, which is localized in axons, becomes activated after axonal injury and regulates a nuclear signaling cascade whose action promotes new axonal growth in the proximal stumps of injured motoneuron axons (Xiong et al., 2010). Here, we describe the role of Wnd in the distal stump of these same neurons.

Contrasting to previous observations in other neurons (Miller et al., 2009; Ghosh et al., 2011), we find that Wnd does not promote degeneration of Drosophila motoneuron axons. Instead,

\footnotetext{
Received July 12, 2011; revised Nov. 27, 2011; accepted Dec. 2, 2011.

Author contributions: X.X. and C.A.C. designed research; X.X. performed research; X.X. analyzed data; X.X. and C.A.C. wrote the paper.

This work was supported by a New Investigator grant (NIRG-09-133497) from the Alzheimer's Association and a grant (NS069844) from the National Institute of Health. We thank Kan Sun, Mary Sprader, and Jennifer Diep for technical assistance, Liqun Luo for UAS-WId ${ }^{5}$ transgenic flies, and Melissa Rolls for helpful discussions. Additionally, we thank the Bloomington Stock Center (Indiana University), the Vienna Drosophila RNAi Center, and the Developmental Studies Hybridoma Bank (University of lowa).

The authors declare no financial conflicts of interest.

Correspondence should be addressed to Catherine A. Collins, Department of Molecular, Cellular and Developmental Biology, University of Michigan, 830 N. University Avenue, Ann Arbor, MI 48109-1048. E-mail: collinca@umich.edu.

DOI:10.1523/JNEUROSCI.3586-11.2012

Copyright $\odot 2012$ the authors $\quad 0270-6474 / 12 / 320610-06 \$ 15.00 / 0$
}

Wnd acts as an inhibitor for Wallerian degeneration of these neurons. Because Wnd becomes acutely upregulated in axons after injury (Xiong et al., 2010), we hypothesized that Wnd promotes an adaptive response in these neurons that is protective against further damage. Using a conditioning lesion assay, we found that motoneuron axons that had been previously injured have an increased resilience to degeneration, and this requires the cell-autonomous function of Wnd and the transcription factor Fos. These findings implicate conserved axonal signaling molecules in a mechanism that controls remarkable plasticity in the axonal degeneration process.

\section{Materials and Methods}

Genetics. The following strains were used in this study: Canton-S (wildtype), BG380-Gal4 (Budnik et al., 1996), m12-Gal4 (P(GAL4)5053A) (Ritzenthaler et al., 2000), $w n d^{1}, w n d^{2}$, and UAS-wnd (Collins et al., 2006), UAS-Fos ${ }^{D N}$ (Fbz) (Eresh et al., 1997), UAS-Bsk(JNK) ${ }^{D N}$ (Weber et al., 2000), and UAS-Wld (Hoopfer et al., 2006). UAS-Dcr2 was gift from Stephan Thor (Linköping University, Linköping Sweden), and UAS-wndRNAi was acquired from the Vienna RNAi Center (Construct ID 13786) (Dietzl et al., 2007). Male larvae were used for all experiments using the BG380-Gal4 driver. For other experiments, larvae of both sexes were used.

Immunocytochemistry. Larvae were dissected in PBS and fixed in either $4 \%$ paraformaldehyde in PBS or Bouin's fixative for 15-30 min, depending on the antibodies used. Antibodies were used at the following dilutions in PBS with $0.3 \%$ Triton X-100 and 5\% normal goat serum: mouse anti-Futsch, 1:100; mouse anti-Brp, 1:50; rabbit anti-GluRIII, 1:1000; rabbit anti-DVGLUT, 1:5000; Cy3-goat anti-HRP (Jackson ImmunoResearch), 1:500; A488-rabbit anti-GFP (from Invitrogen), 1:1000. For secondary antibodies, $\mathrm{Cy} 3$ and A488-conjugated goat anti-rabbit and anti-mouse (Invitrogen) were used at 1:1000.

Nerve crush assay. The segmental nerves of third instar larvae were subjected to nerve crush injury as previously described (Xiong et al., 2010). For the conditioning lesion assay, larvae were injured at segment A4 for the first injury and at segment A2 for the second injury. The larvae were kept at $25^{\circ} \mathrm{C}$ on a grape plate after or between injuries.

Imaging and quantification. Confocal images were collected at room temperature on an Improvision spinning disk confocal system 

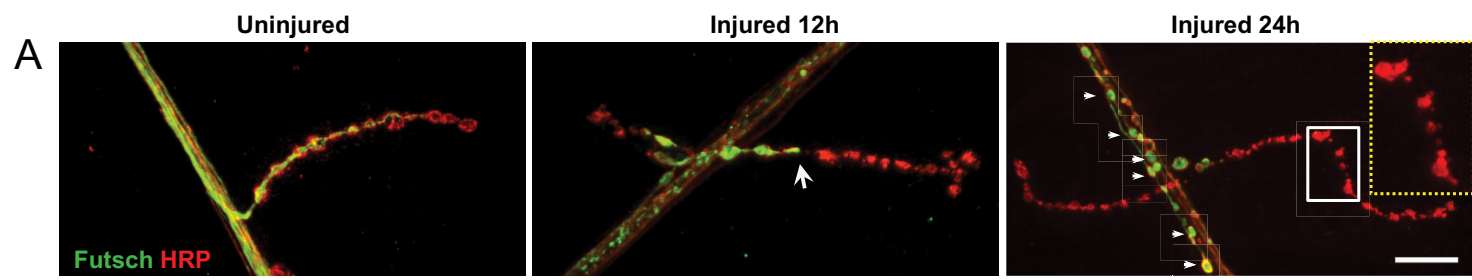

$\mathrm{B}$
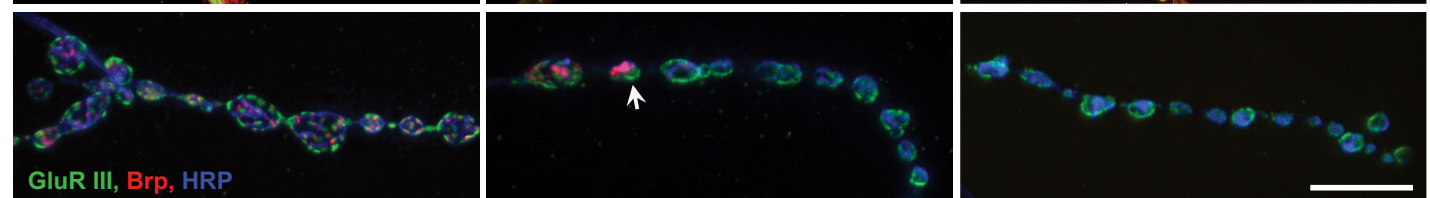

C
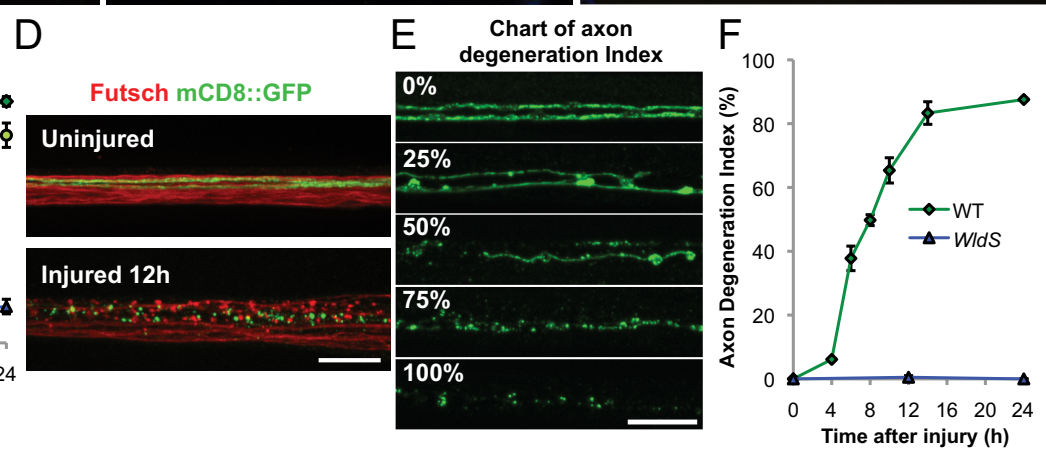

Figure 1. Characterization of degeneration at the Drosophila NMJ and in motoneuron axons after injury. $A$, The axonal membrane (red) at muscle 4 NMJ synapses is labeled by immunostaining with anti-HRP antibodies. The MAP1B homolog Futsch (green) disappears most rapidly from distal boutons and concentrates within proximal boutons (12 h, arrow). By $24 \mathrm{~h}$, Futsch has completely disappeared from boutons and forms sphere-shaped fragments in segmental nerves (arrowheads) and HRP staining becomes discontinuous (inset). $\boldsymbol{B}$, Presynaptic active zones, stained by anti-Brp antibodies (red) also disappear from NMJ boutons in a distal-to-proximal manner ( $12 \mathrm{~h}$, arrow). Postsynaptic receptors, detected by GluRIll staining (green) become diffuse but do not disappear ( 24 h). C, The time course of NMJ degeneration was quantified by measuring the length of muscle 4 NMJ nerve terminus (defined by HRP staining), which does not contain Futsch (as described in Materials and Methods). The onset of degeneration is significantly delayed at the lower temperature (dotted line), and inhibited by expression of the WIdS mutation (blue line). $\boldsymbol{D}$, In segmental nerves distal to the injury site, Futsch (red) and mCD8::GFP-labeled single axons, using the m12-Gal4 driver (green), are extensively fragmented by $12 \mathrm{~h}$ after injury. Nerves are oriented with the side proximal to the injury on the left. $E, F$, To quantify the time course of axonal degeneration, axons were scored by researchers blind to genotype using a scale ranging from completely continuous ( $0 \%$ ) to continuous with varicosities (25\%) to partially discontinuous (50\%) to mostly fragmented with a few segments of continuity (75\%) to completely fragmented (100\%). Scale bars, $25 \mu \mathrm{m}$.

(PerkinElmer). Similar settings were used to collect all compared genotypes and conditions.

To quantify the extent of degeneration of the neuromuscular junction (NMJ), we measured disappearance of Futsch from NMJ boutons. This MAP1B homolog binds stable microtubules, which in uninjured axons extend through all but the most terminal boutons (Hummel et al., 2000; Roos et al., 2000). The NMJ degeneration index is defined as the length of NMJ without Futsch staining divided by the total length of the nerve terminal, which is determined by HRP staining [which is coincident with postsynaptic markers (data not shown)]. Total lengths were measured using the simple neurite tracer plugin of ImageJ software. All quantifications shown represent the average from $>40$ NMJs from $>10$ animals.

To quantify axonal degeneration, we scored while blind to genotype the fragmentation of m12-Gal4, UAS-mCD8:GFP labeled axons within segmental nerves according to one of five categories (described in Fig. $1 E)$. All measurements indicate the average from $>100$ axons.

\section{Results}

Wallerian degeneration at the Drosophila NMJ and in motoneuron axons

The facile genetics and highly characterized neuroanatomy of Drosophila make it a powerful model organism to study degeneration mechanisms. We have recently developed a nerve crush assay in which the segmental nerves of third instar larvae are pinched with forceps, resulting in acute transection of motoneuron and sensory neuron axons (Xiong et al., 2010). After nerve crush, Wallerian degeneration can be observed in both axons and nerve terminals (NMJs) distal to the site of transection (Fig. 1).

At the NMJ, synaptic boutons from injured neurons became disconnected from one another, and then devoid of presynaptic proteins, including cytoskeletal proteins, active zone components and synaptic vesicles components (Fig. $1 A-C$ and data not shown). This disappearance occurred in a distal-to-proximal manner: at $12 \mathrm{~h}$ after injury, the most distal boutons were fragmented and empty, and by $24 \mathrm{~h}$ after injury, all presynaptic components had completely disappeared from the NMJ. We exploited this distal-to-proximal disappearance to quantify the extent of NMJ degeneration over a time course (Fig. 1C) by measuring the length of the NMJ nerve terminal that did not contain the MAP1B homolog Futsch (described in Materials and Methods, above). The disappearance of Futsch is synchronous with other presynaptic markers (data not shown). In contrast, postsynaptic proteins did not disappear, even $24 \mathrm{~h}$ after injury (Fig. $1 B$ ). This process is quite distinct from the way in which the NMJ becomes dismantled during metamorphosis (Liu et al., 2010).

Within segmental nerves, endogenous Futsch became fragmented into spherical structures within $12 \mathrm{~h}$ after injury (Fig. $1 D)$, which were less abundant by $24 \mathrm{~h}$, suggesting clearance (data not shown). To quantify axonal degeneration, we assessed the continuity of single motoneuron axons, labeled by expression of mCD8::GFP using the Gal4/UAS system (Fig. $1 D, E$ ). For both axonal and synaptic degeneration, we observed a temperaturedependent lag phase followed by a rapid degeneration phase (Fig. $1 C)$ that is synchronous throughout the axon.

Both axonal and synaptic degeneration were inhibited by expression of the mouse WldS mutant protein (Fig. 1C,F), emphasizing that the degeneration program shares mechanistic sim- 

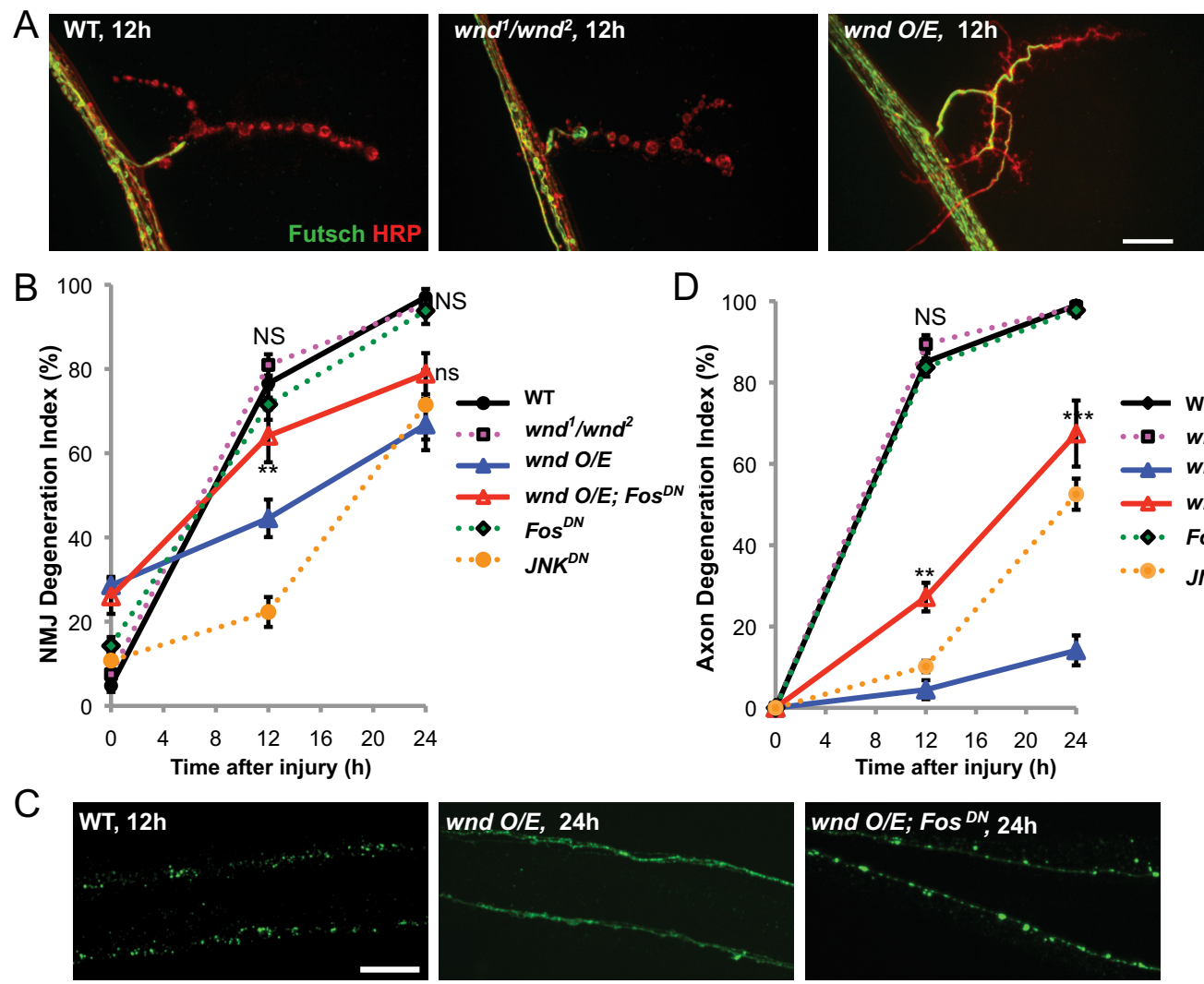

D
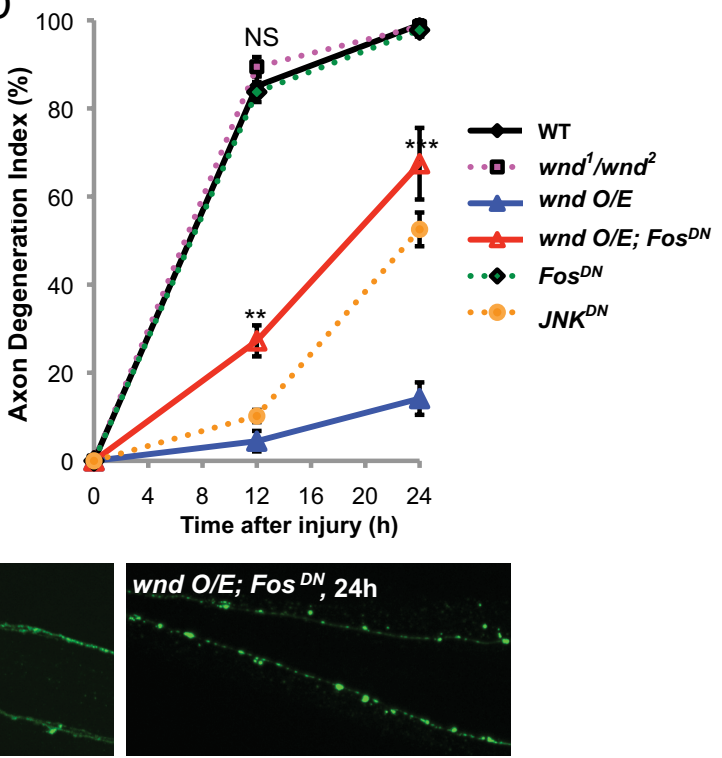

Figure 2. The Wallenda/DLK kinase inhibits Wallerian degeneration. $A$, Representative muscle 4 NMJs are shown for wild-type (Canton S), wnd mutant (wnd ${ }^{1} /$ wnd $^{2}$ ), and overexpressed wnd (BG380-Gal4; UAS-wnd/+) $12 \mathrm{~h}$ after injury. B, Quantification of the NMJ degeneration index (defined by absence of Futsch staining) over a time course for different genotypes. wnd mutant (magenta) and $F o S^{D N}$ (BG380;;UAS-Fos ${ }^{D N} /+$; green) degenerate with a similar time course as wild-type axons. NS, No significant difference from wild-type. However, overexpression of wnd (blue) dramatically delays degeneration. This inhibition of degeneration is strongly reduced when Fos ${ }^{D N}$ is coexpressed (BG380;UAS-wnd/+; UAS-Fos ${ }^{D N} /+;$ red). ${ }^{* *} p<0.01$, compared with wnd $0 / E$. An additional phenotype of wnd overexpression is a reduction of Futsch in terminal boutons, which results in a higher degeneration index in uninjured animals. However, this phenotype is not suppressed by coexpression of Fos ${ }^{D N}$. C, Representative images of single axons in wild-type axons (UAS-mCD8::GFP/+; m12-Gal4/+) that degenerated within $12 \mathrm{~h}$ of injury, compared with axons that remained intact $24 \mathrm{~h}$ after injury when wnd was overexpressed (UAS-wnd/UAS-mCD8::GFP; $\mathrm{m} 12-$ Gal4/+). This protection from degeneration is inhibited by coexpression of Fos ${ }^{D N}$ (UAS-wnd/UAS-mCD8::GFP; m12-Gal4/UAS-Fos ${ }^{D N}$ ). D, Quantification of axon degeneration in different genotypes. ${ }^{* *} p<0.01,{ }^{* * *} p<0.001$ compared with wnd 0/E. Scale bars, $25 \mu \mathrm{m}$.

ilarities from vertebrates to invertebrates (Hoopfer et al., 2006; MacDonald et al., 2006). Based on these observations, motoneuron axons and synapses in Drosophila larvae can be used as a model system to study the cellular mechanisms of degeneration after injury.

\section{The Wnd/DLK kinase protects axons from}

Wallerian degeneration

A previous study in mice and Drosophila olfactory neurons has implicated a role for the Wallenda/DLK kinase in promoting the process of Wallerian degeneration (Miller et al., 2009). However, our assay for Wallerian degeneration in Drosophila motoneurons reveals no requirement for Wnd in either synaptic or axonal degeneration after injury (Fig. $2 A, B, D$ ). In contrast, we observed that overexpression of $w n d$ caused a dramatic delay in both synaptic and axonal degeneration (Fig. 2). When wnd was overexpressed, synaptic boutons and axons remained significantly intact (33.2\% and $85.8 \%$, respectively) even $24 \mathrm{~h}$ after injury; in comparison, wild-type synapses and axons had completely degenerated in this time. Overexpression of a kinase-dead version of $w n d$ had no effect on degeneration (data not shown). These observations suggest that the action of this kinase has a protective effect in motoneuron axons and synapses.

Previous studies indicate that Wnd regulates synaptic growth and the initiation of new axonal growth after injury via a nuclear signaling cascade (Collins et al., 2006; Yan et al., 2009; Xiong et al., 2010). Similar to previously described wnd overexpression phenotypes (Collins et al., 2006; Xiong et al., 2010), we find that the protection from degeneration by Wnd is inhibited by overexpression of a dominant-negative Fos transgene $\left(F_{0 s}{ }^{D N}\right)$ in motoneurons (Fig. $2 B-D$ ). This finding suggests that the protective action of Wnd involves changes in gene expression.

Wnd/DLK is thought to function as an upstream regulator of the JNK MAP kinase (Nihalani et al., 2001; Hirai et al., 2002; Collins et al., 2006; Xiong et al., 2010; Ghosh et al., 2011; Nix et al., 2011). While JNK is required for nuclear signaling by Wnd (Xiong et al., 2010), we could not address the role of JNK in protection by Wnd, because inhibition of JNK alone also led to protection from axonal degeneration (Fig. $2 B, D$, yellow line). This observation parallels observations in vertebrate axons (Miller et al., 2009; Barrientos et al., 2011; Yoshimura et al., 2011). However, because mutation or knockdown of wnd did not delay degeneration, we hypothesize that additional and independent regulators of JNK may function in promoting degeneration of the distal stump after injury.

\section{A previous injury protects axons from}

Wallerian degeneration

Our findings indicated that activation of Wnd signaling in motoneurons could increase their resiliency to axonal degeneration. 
A

conditioning lesion

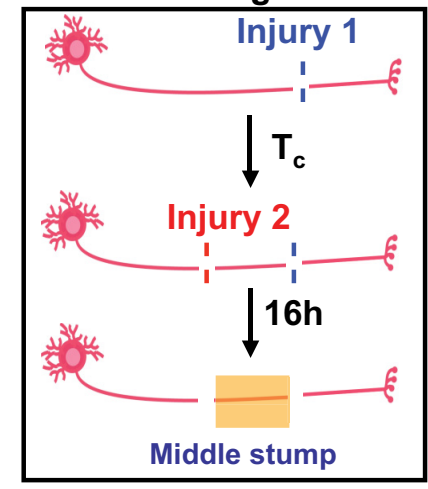

C
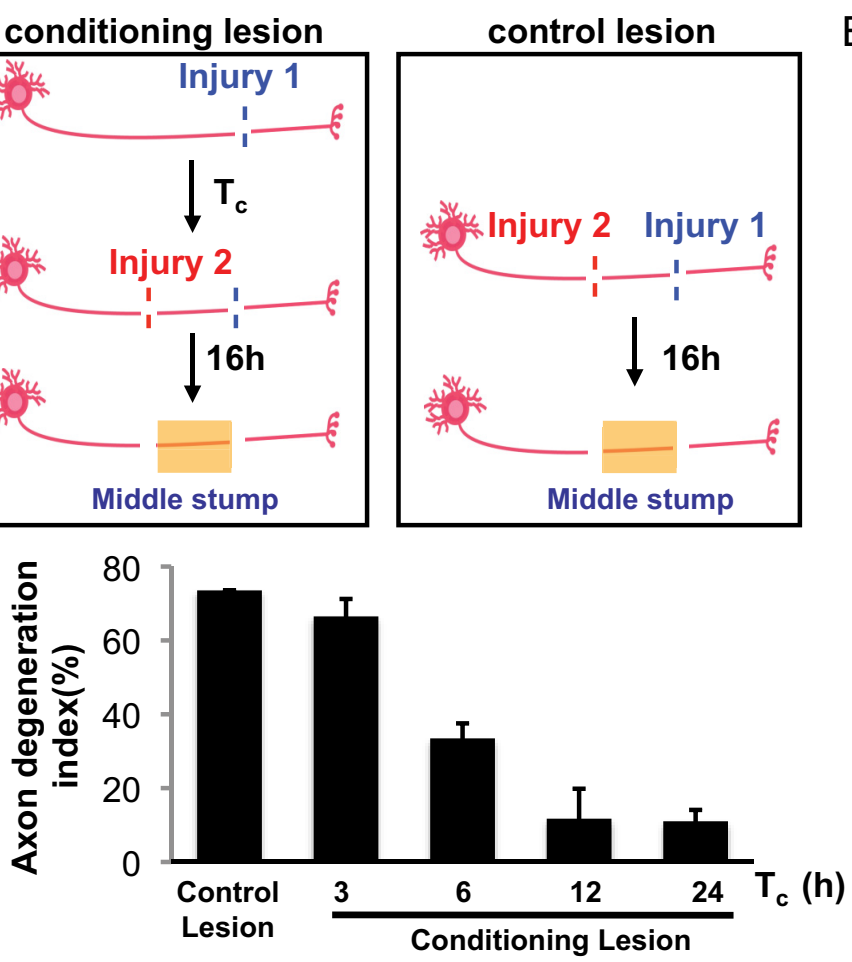

$B$
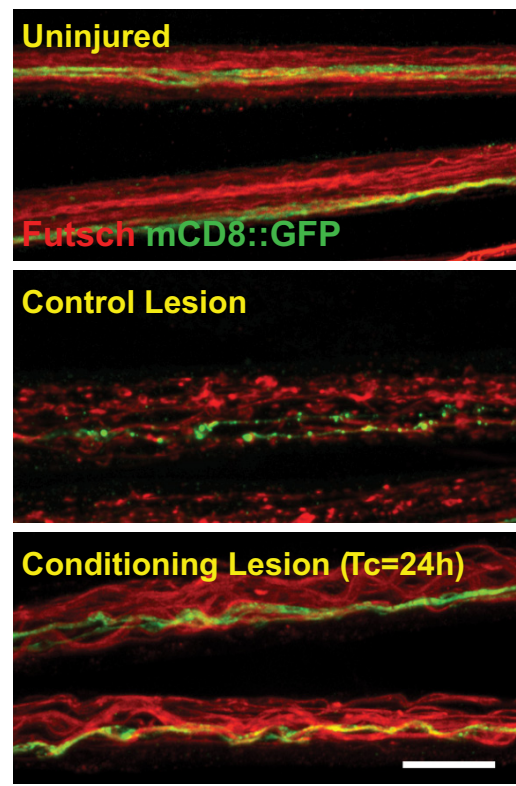

Figure 3. A conditioning lesion protects axons from degeneration after a second injury. $\boldsymbol{A}$, Schematic of the conditioning lesion and control lesion experiments. A conditioning lesion was done at injury site 1 (see Materials and Methods) for a variable period of time, $T_{C^{\prime}}$ before the second injury at site 2 , which was within the proximal stump of the previously injured axon. $\boldsymbol{B}$, Uninjured axons or middle stump axons in control and conditioning lesion were stained for Futsch (red) and GFP (green). Single axons, labeled by driving UAS-mCD8::GFP expression with $m 12-G a / 4$, and endogenous Futsch were fragmented at $16 \mathrm{~h}$ after the control lesion, but intact after the conditioning lesion. Scale bar, $25 \mu \mathrm{m}$. C, Quantification of the axon degeneration index, measured at $16 \mathrm{~h}$ after the second injury. For the conditioning lesion, the time between the first and second injuries, $\mathrm{T}_{{ }^{\prime}}$ was varied.

While Wnd is normally kept at very low levels in larval motoneurons (Collins et al., 2006), recent studies suggest that Wnd protein becomes upregulated and activated in axons after axonal injury (Xiong et al., 2010). These observations led us to hypothesize that endogenous Wnd protein may promote a protective response in neurons that have sustained an injury. Studies in vertebrate neurons suggest that a conditioning lesion can dramatically alter the response of a neuron to subsequent injuries (Hoffman, 2010). We hypothesized that Wnd function, which becomes activated by axonal injury in the PNS, allows for neurons that have been previously injured to have an increased resiliency to degeneration. To test this, we conducted a conditioning lesion assay (Fig. $3 A$ ) in which the proximal stump after an initial injury was injured a second time. Degeneration was then measured in the middle stump distal to the second injury site. We found that if an axon had been injured at least $6 \mathrm{~h}$ previously, then degeneration after the second injury was significantly delayed (Fig. $3 B, C$ ). Similar to the effect of conditioning lesion on regeneration in vertebrate neurons (Hoffman, 2010), the protective effect of the conditioning lesion required time; in this case, $6 \mathrm{~h}$ to reach partial effect and $12 \mathrm{~h}$ to reach maximal effect (Fig. 3C). We conclude that a conditioning injury to axons induces changes in the cell that lead to protection of axons from degeneration after future injuries.

The Wallenda signaling cascade mediates an adaptive response in neurons

Importantly, the time requirement for the protective effect of the conditioning lesion matches other cellular events that have been previously characterized in this nerve crush injury assay (Xiong et al., 2010). For instance, phosphorylated JNK is induced in the cell body $\sim 6 \mathrm{~h}$ after injury, and transcriptional changes can be detected in the nucleus $\sim 8 \mathrm{~h}$ after injury. All of these events are preceded by an increase in the levels of Wnd protein within $4 \mathrm{~h}$ of injury. We therefore tested whether the protective effect of the conditioning lesion requires Wnd function (Fig. 4). When we examined axons in the middle stump after the conditioning lesion assay, axons from $w n d^{1} / w n d^{2}$ mutants degenerated similarly to axons that had received the control (unconditioned) double lesion (Fig. 4A), suggesting that the conditioning lesion had no effect in wnd mutants. Furthermore, expression of wnd RNAi or Fos ${ }^{D N}$ specifically in the mCD8::GFP-labeled motoneurons (Fig. $4 B, C)$ abolished the protective effect of the conditioning lesion (Fig. 4C). The neighboring axons that did not express the transgenes were still protected from degeneration (Fig. $4 B$ ), demonstrating the cell autonomy of the pathway. We conclude that a signaling cascade downstream of the Wnd kinase, which becomes activated by axonal injury, promotes a protective response in axons that inhibits Wallerian degeneration after injury (Fig. $4 D$ ).

\section{Discussion}

Our findings suggest that the Wnd kinase plays a protective role in Drosophila motoneuron axons. Wnd protein is normally kept at a low level in these axons, but becomes rapidly induced by axonal injury (Xiong et al., 2010). This leads to activation of a nuclear signaling cascade that promotes an increased resiliency of axons to degeneration after a second injury. The requirement for Wnd in the protective effect of a conditioning lesion leads us to 

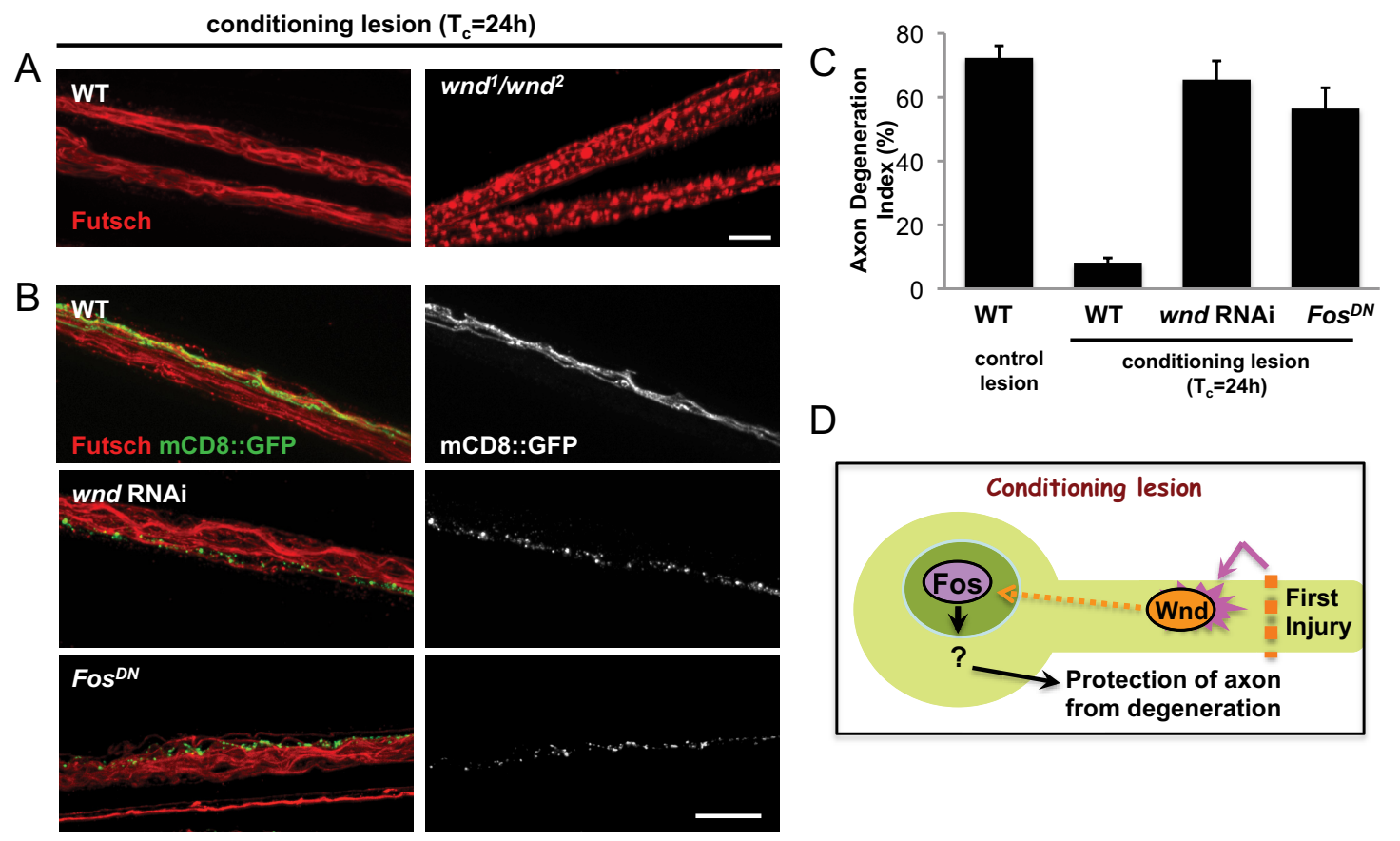

D

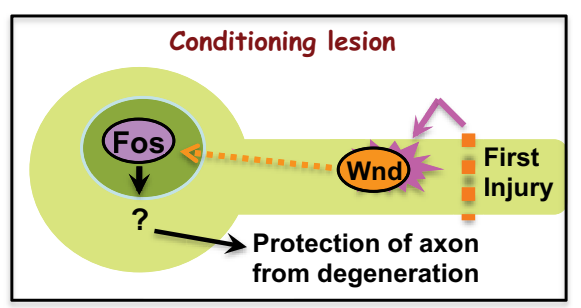

Figure 4. The protective effect of the conditioning lesion requires Wallenda. $A$, Staining for endogenous Futsch in the segmental nerves of axons treated with conditioning lesion, as outlined in Figure 3A. In wnd ${ }^{1} /$ wnd $^{2}$ mutants, endogenous Futsch was fragmented similarly to control, singly injured axons (Fig. 1C). B, The m12-Gal4 driver line was used to drive expression of wnd-RNAi (along with UAS-DCr2) or Fos ${ }^{D N}$ in the GFP-labeled axons. The $\mathrm{mCD} 8:$ :GFP labeled axons are shown alone in grayscale (right). All axons were treated with a conditioning lesion with a $\mathrm{T}_{\mathrm{c}}$ of $24 \mathrm{~h}$, and the middle stump is shown $16 \mathrm{~h}$ after the second injury. Scale bars, $25 \mu \mathrm{m}$. C, Quantification of the axon degeneration index indicates that expression of wnd RNAi or Fos ${ }^{D N}$ nearly completely abolishes the protective effect of the conditioning lesion. $\boldsymbol{D}$, Model for the role of Wallenda, which becomes activated in axons by injury and mediates a signaling cascade that requires time and the Fos transcription factor. The downstream cellular changes that alter resiliency to degeneration remain to be determined.

propose that Wnd regulates a stress response pathway that allows neurons to adapt to axonal injury.

A pathway that inhibits axonal degeneration would be very advantageous in instances where the integrity of the axon is compromised but not completely lost, such as anoxia, loss of myelination, or defects in axonal transport. Such problems are a concern not only for neuronal injuries, but also neurodegenerative disorders, whose onset may be influenced by neuronal stress (Mattson and Magnus, 2006). Along these lines, mutations that lead to activation of Wnd/DLK signaling can suppress a synaptic retraction phenotype in Drosophila (Massaro et al., 2009).

The conditioning lesion is classically studied for its role in facilitating axonal regeneration, although it has been noted that a conditioning lesion can also attenuate degeneration of motoneurons in a rat model of ALS (Franz et al., 2009). In vertebrates, a conditioning injury of axons in the PNS induces widespread cellular changes, including chromatolysis and changes in gene expression, translation, trafficking, cytoskeleton, and physiology (Cragg, 1970; Hoffman, 2010). Studies in multiple model organisms have already implicated Wnd/DLK in nuclear signaling (Yan et al., 2009; Xiong et al., 2010; Ghosh et al., 2011), translation (Yan et al., 2009), axonal transport (Horiuchi et al., 2007), and cytoskeletal dynamics (Eto et al., 2010; Bounoutas et al., 2011; Hirai et al., 2011); hence, this kinase may function as an upstream regulator of multiple downstream responses to axonal injury. The exact cellular events that lead to protection from degeneration and their relationship to the regenerative response remain to be determined.

Importantly, the action of Wnd/DLK is not always beneficial. In contrast to motoneuron axons, Wnd is unable to protect olfactory neuron axons in the adult. Instead, Wnd plays a modest role in promoting degeneration in these neurons (Miller et al.,
2009). Also in contrast, the vertebrate homolog DLK plays a prodegenerative role in DRG neurons, both after injury (Miller et al., 2009) and after nerve growth factor withdrawal (Ghosh et al., 2011). The distinct downstream outcomes may depend on distinct features of downstream signaling events in different cell types, developmental contexts, and cellular location. For instance, the protective role of Wnd requires time, the neuronal cell body, and downstream gene expression, while the distal stump of injured axon would be unable to receive the benefits of a nuclear signaling cascade induced by injury. However, even the nuclear signaling cascade in the proximal stump is not always beneficial: in cultured DRG neurons, DLK promotes apoptosis after nerve growth factor withdrawal (Ghosh et al., 2011). This negative outcome of DLK action shares mechanistic similarities with the beneficial outcome of Wnd for regeneration in that both depend on retrograde signaling to the nucleus after an axonal stimulus (Xiong et al., 2010; Ghosh et al., 2011).

Similar to previous studies (Miller et al., 2009; Barrientos et al., 2011; Yoshimura et al., 2011), our findings suggest that JNK plays a pro-degenerative role in Drosophila motoneuron axons; however, this action may occur independently of Wnd regulation. Indeed, a Wnd-independent pool of phosphorylated JNK has been described in Drosophila axons (Xiong et al., 2010). While JNK plays an important role in the regenerative response to injury (Xiong et al., 2010), it is not clear whether JNK functions in the injury-induced protection from degeneration. An equally likely candidate mediator, based on studies in C. elegans (Nakata et al., 2005; Nix et al., 2011), is the other stress-activated MAPK, p38. Future characterization of the mechanism of Wnd/DLK signaling, including the cofactors and effectors for its different functions in different contexts, will be important for delineating the molecular differences between positive and negative responses to axonal damage. 


\section{Notes}

Supplemental material for this article is available at www.mcdb.lsa. umich.edu/labs/collins/files/suppfigures112.docx. Figure 1: Wnd does not protect all neurons from degeneration after injury. This figure shows that overexpression in adult olfactory neurons and larval class IV sensory neurons does not inhibit Wallerian degeneration after injury. Figure 2: Additional manipulations in JNK, p38b, and Jun that do not lead to protection from degeneration. This material has not been peer reviewed.

\section{References}

Barrientos SA, Martinez NW, Yoo S, Jara JS, Zamorano S, Hetz C, Twiss JL, Alvarez J, Court FA (2011) Axonal degeneration is mediated by the mitochondrial permeability transition pore. J Neurosci 31:966-978.

Bounoutas A, Kratz J, Emtage L, Ma C, Nguyen KC, Chalfie M (2011) Microtubule depolymerization in Caenorhabditis elegans touch receptor neurons reduces gene expression through a p38 MAPK pathway. Proc Natl Acad Sci U S A 108:3982-3987.

Budnik V, Koh YH, Guan B, Hartmann B, Hough C, Woods D, Gorczyca M (1996) Regulation of synapse structure and function by the Drosophila tumor suppressor gene $\mathrm{dlg}$. Neuron 17:627-640.

Coleman M (2005) Axon degeneration mechanisms: commonality amid diversity. Nat Rev Neurosci 6:889-898.

Collins CA, Wairkar YP, Johnson SL, DiAntonio A (2006) Highwire restrains synaptic growth by attenuating a MAP kinase signal. Neuron 51:57-69.

Cragg BG (1970) What is the signal for chromatolysis? Brain Res 23:1-21.

Dietzl G, Chen D, Schnorrer F, Su KC, Barinova Y, Fellner M, Gasser B, Kinsey K, Oppel S, Scheiblauer S, Couto A, Marra V, Keleman K, Dickson BJ (2007) A genome-wide transgenic RNAi library for conditional gene inactivation in Drosophila. Nature 448:151-156.

Eresh S, Riese J, Jackson DB, Bohmann D, Bienz M (1997) A CREB-binding site as a target for decapentaplegic signalling during Drosophila endoderm induction. EMBO J 16:2014-2022.

Eto K, Kawauchi T, Osawa M, Tabata H, Nakajima K (2010) Role of dual leucine zipper-bearing kinase (DLK/MUK/ZPK) in axonal growth. Neurosci Res 66:37-45.

Franz CK, Quach ET, Krudy CA, Federici T, Kliem MA, Snyder BR, Raore B, Boulis NM (2009) A conditioning lesion provides selective protection in a rat model of amyotrophic lateral sclerosis. PLoS One 4:e7357.

Ghosh AS, Wang B, Pozniak CD, Chen M, Watts RJ, Lewcock JW (2011) DLK induces developmental neuronal degeneration via selective regulation of proapoptotic JNK activity. J Cell Biol 194:751-764.

Ghosh-Roy A, Wu Z, Goncharov A, Jin Y, Chisholm AD (2010) Calcium and cyclic AMP promote axonal regeneration in Caenorhabditis elegans and require DLK-1 kinase. J Neurosci 30:3175-3183.

Hammarlund M, Nix P, Hauth L, Jorgensen EM, Bastiani M (2009) Axon regeneration requires a conserved MAP kinase pathway. Science 323: 802-806.

Hirai S, Kawaguchi A, Hirasawa R, Baba M, Ohnishi T, Ohno S (2002) MAPK-upstream protein kinase (MUK) regulates the radial migration of immature neurons in telencephalon of mouse embryo. Development 129:4483-4495.

Hirai S, Banba Y, Satake T, Ohno S (2011) Axon formation in neocortical neurons depends on stage-specific regulation of microtubule stability by the dual leucine zipper kinase-c-jun $N$-terminal kinase pathway. J Neurosci 31:6468-6480.

Hoffman PN (2010) A conditioning lesion induces changes in gene expres- sion and axonal transport that enhance regeneration by increasing the intrinsic growth state of axons. Exp Neurol 223:11-18.

Hoopfer ED, McLaughlin T, Watts RJ, Schuldiner O, O’Leary DD, Luo L (2006) Wlds protection distinguishes axon degeneration following injury from naturally occurring developmental pruning. Neuron 50:883895.

Horiuchi D, Collins CA, Bhat P, Barkus RV, Diantonio A, Saxton WM (2007) Control of a kinesin-cargo linkage mechanism by JNK pathway kinases. Curr Biol 17:1313-1317.

Hummel T, Krukkert K, Roos J, Davis G, Klämbt C (2000) Drosophila Futsch/22C10 is a MAP1B-like protein required for dendritic and axonal development. Neuron 26:357-370.

Itoh A, Horiuchi M, Bannerman P, Pleasure D, Itoh T (2009) Impaired regenerative response of primary sensory neurons in ZPK/DLK gene-trap mice. Biochem Biophys Res Commun 383:258-262.

Liu Z, Chen Y, Wang D, Wang S, Zhang YQ (2010) Distinct presynaptic and postsynaptic dismantling processes of Drosophila neuromuscular junctions during metamorphosis. J Neurosci 30:11624-11634.

MacDonald JM, Beach MG, Porpiglia E, Sheehan AE, Watts RJ, Freeman MR (2006) The Drosophila cell corpse engulfment receptor Draper mediates glial clearance of severed axons. Neuron 50:869-881.

Massaro CM, Pielage J, Davis GW (2009) Molecular mechanisms that enhance synapse stability despite persistent disruption of the spectrin/ ankyrin/microtubule cytoskeleton. J Cell Biol 187:101-117.

Mattson MP, Magnus T (2006) Ageing and neuronal vulnerability. Nat Rev Neurosci 7:278-294

Miller BR, Press C, Daniels RW, Sasaki Y, Milbrandt J, DiAntonio A (2009) A dual leucine kinase-dependent axon self-destruction program promotes Wallerian degeneration. Nat Neurosci 12:387-389.

Nakata K, Abrams B, Grill B, Goncharov A, Huang X, Chisholm AD, Jin Y (2005) Regulation of a DLK-1 and p38 MAP kinase pathway by the ubiquitin ligase RPM-1 is required for presynaptic development. Cell 120:407-420.

Nihalani D, Meyer D, Pajni S, Holzman LB (2001) Mixed lineage kinasedependent JNK activation is governed by interactions of scaffold protein JIP with MAPK module components. EMBO J 20:3447-3458.

Nix P, Hisamoto N, Matsumoto K, Bastiani M (2011) Axon regeneration requires coordinate activation of $\mathrm{p} 38$ and JNK MAPK pathways. Proc Natl Acad Sci U S A 108:10738-10743.

Ritzenthaler S, Suzuki E, Chiba A (2000) Postsynaptic filopodia in muscle cells interact with innervating motoneuron axons. Nat Neurosci 3:1012-1017.

Roos J, Hummel T, Ng N, Klämbt C, Davis GW (2000) Drosophila Futsch regulates synaptic microtubule organization and is necessary for synaptic growth. Neuron 26:371-382.

Weber U, Paricio N, Mlodzik M (2000) Jun mediates Frizzled-induced $\mathrm{R} 3 / \mathrm{R} 4$ cell fate distinction and planar polarity determination in the Drosophila eye. Development 127:3619-3629.

Xiong X, Wang X, Ewanek R, Bhat P, Diantonio A, Collins CA (2010) Protein turnover of the Wallenda/DLK kinase regulates a retrograde response to axonal injury. J Cell Biol 191:211-223.

Yan D, Wu Z, Chisholm AD, Jin Y (2009) The DLK-1 kinase promotes mRNA stability and local translation in C. elegans synapses and axon regeneration. Cell 138:1005-1018.

Yoshimura K, Ueno M, Lee S, Nakamura Y, Sato A, Yoshimura K, Kishima H, Yoshimine T, Yamashita T (2011) C-jun N-terminal kinase induces axonal degeneration and limits motor recovery after spinal cord injury in mice. Neurosci Res 71:266-277. 